\title{
PRESERVING PROPERTIES OF SUBORDINATION AND SUPERORDINATION OF ANALYTIC FUNCTIONS ASSOCIATED WITH A FRACTIONAL DIFFERINTEGRAL OPERATOR
}

\author{
JAMAL M. SHENAN
}

\begin{abstract}
In this paper, we obtain some subordination and superordination-preserving results of analytic functions associated with the fractional differintegral operator $U_{0, z}^{\alpha, \beta, \gamma}$. Sandwich-type result involving this operator is also derived.
\end{abstract}

\section{Introduction}

Let $H(U)$ be the class of functions analytic in $U=\{z: z \in C$ and $|z|<1\}$ and $H[a, k]$ be the subclass of $H(U)$ consisting of functions of the form $f(z)=a+a_{k} z^{k}+a_{k+1} z^{k+1}+\cdots$, with $H_{0} \equiv H[0,1]$ and $H \equiv H[1,1]$.

Let $A_{p}$ denote the class of functions of the form

$$
f(z)=z^{p}+\sum_{n=1}^{\infty} a_{n+p} z^{n+p}(p, \in \mathbb{N}=\{1,2,3, \ldots\} ; z \in U),
$$

which are analytic in the open unit $\operatorname{disk} U$.

Let $f$ and $F$ be members of $H(U)$, the function $f(z)$ is said to be subordinate to $F(z)$, or $F(z)$ is said to be superordinate to $f(z)$, if there exists a function $w(z)$ analytic in $U$ with $w(0)=0$ and $|w(z)|<1(z \in U)$, such that $f(z)=F(w(z))$. In such a case we write $f(z) \prec F(z)$. In particular, if $F$ is univalent, then $f(z) \prec F(z)$ if and only if $f(0)=F(0)$ and $f(U) \subset F(U)$ (see $[5,6])$.

Let $\Psi: C^{2} \times U \rightarrow C$ and let $h$ be univalent in $U$. If $p$ is analytic in $U$ and satisfies the first order differential subordination

$$
\Psi\left(p(z), z p^{\prime}(z) ; z\right) \prec h(z)(z \in U)
$$

then $p$ is called a solution of the differential subordination (1.2).

Received December 20, 2012, accepted May 17, 2013.

2010 Mathematics Subject Classification. 30C45.

Key words and phrases. Analytic function, multivalent function, fractional differintegral operator, subordination, Superordination. 
The univalent function $q$ is called a dominant solutions of the differential subordination (1.2) if $p \prec q$ for all $p$ satisfying (1.2). A dominant $\tilde{q}$ that satisfies $\tilde{q} \prec q$ for all dominants $q$ of (1.2) is said to be the best dominant of (1.2).

Similarly, let $\Phi: C^{2} \times U \rightarrow C$ and let $h$ be univalent in $U$. If $p$ is analytic in $U$ and satisfies the first order differential superordination

$$
h(z) \prec \Phi\left(p(z), z p^{\prime}(z) ; z\right)(z \in U)
$$

then $p$ is called a solution of the differential superordination (1.3).

The univalent function $q$ is called a subordinant solutions of the differential superordination (1.3) if $q \prec p$ for all $p$ satisfying (1.3). A subordinant $\tilde{q}$ that satisfies $q<\tilde{q}$ for all subordinant $q$ of (1.3) is said to be the best subordinant. ( see the monograph by Miller and Mocanu [7], and $[8])$.

We recall the definitions of the fractional derivative and integral operators introduced and studied by Saigo (cf. [14], [15]).

Definition 1. Let $\alpha>0$ and $\beta, \gamma \in R$, then the generalized fractional integral operator $\mathrm{I}_{0, z}^{\alpha, \beta, \gamma}$ of order $\alpha$ of a function $f(z)$ is defined by

$$
\mathrm{I}_{0, z}^{\alpha, \beta, \gamma} f(z)=\frac{z^{-\alpha-\beta}}{\Gamma(\alpha)} \int_{0}^{z}(z-t)_{2}^{\alpha-1} F_{1}\left(\alpha+\beta,-\gamma ; \alpha ; 1-\frac{t}{z}\right) f(t) d t,
$$

where the function $f(z)$ is analytic in a simply-connected region of the $z$-plane containing the origin and the multiplicity of $(z-t)^{(\alpha-1)}$ is removed by requiring $\log (z-t)$ to be real when $(z-t)>0$ provided further that

$$
f(z)=O\left(|z|^{\varepsilon}\right), z \rightarrow 0 \quad \text { for } \varepsilon>\max (0, \beta-\gamma)-1
$$

Definition 2. Let $0 \leq \alpha<1$ and $\beta, \gamma \in R$, then the generalized fractional derivative operator $J_{0, z}^{\alpha, \beta, \gamma}$ of order $\alpha$ of a function $f(z)$ defined by

$$
\begin{aligned}
J_{0, z}^{\alpha, \beta, \gamma} f(z) & =\frac{1}{\Gamma(1-\alpha)} \frac{d}{d z}\left[z^{\alpha-\beta} \int_{0}^{z}(z-t)_{2}^{-\alpha} F_{1}\left(\beta-\alpha, 1-\gamma ; 1-\alpha ; 1-\frac{t}{z}\right) f(t) d t\right], \\
& =\frac{d^{n}}{d z^{n}} J_{0, z}^{\alpha-n, \beta, \gamma} f(z) \quad(n \leq \alpha<n+1 ; n \in N),
\end{aligned}
$$

where the function $f(z)$ is analytic in a simply-connected region of the $z$-plane containing the origin, with the order as given in (1.5) and multiplicity of $(z-t)^{\alpha}$ is removed by requiring $\log (z-t)$ to be real when $(z-t)>0$.

Note that

$$
I_{0, z}^{\alpha,-\alpha, \gamma} f(z)=D_{z}^{-\alpha} f(z)(\alpha>0)
$$


and

$$
J_{0, z}^{\alpha, \alpha, \gamma} f(z)=D_{z}^{\alpha} f(z)(0 \leq \alpha<1),
$$

where $D_{z}^{-\alpha} f(z)$ and $D_{z}^{\alpha} f(z)$ are respectively the well known Riemann-Liouvill fractional integral and derivative operators (cf. [10] and [11], see also [16]).

Definition 3. For real number $\alpha(-\infty<\alpha<1)$ and $\beta(-\infty<\beta<1)$ and a positive real number $\gamma$, the fractional operator $U_{0, z}^{\alpha, \beta, \gamma}{ }_{-}: A_{p} \rightarrow A_{p}$ is defined in terms of $J_{0, z}^{\alpha, \beta, \gamma}$ and $\mathrm{I}_{0, z}^{\alpha, \beta, \gamma}$ by (see [9] and [4])

$$
U_{0, z}^{\alpha, \beta, \gamma} f(z)=z^{p}+\sum_{n=1}^{\infty} \frac{(1+p)_{n}(1+p+\gamma-\beta)_{n}}{(1+p-\beta)_{n}(1+p+\gamma-\alpha)_{n}} a_{n+p} z^{n+p},
$$

which for $f(z) \neq 0$ may be written as

$$
U_{0, z}^{\alpha, \beta, \gamma} f(z)= \begin{cases}\frac{\Gamma(1+p-\beta) \Gamma(1+p+\gamma-\alpha)}{\Gamma(1+p) \Gamma(1+p+\gamma-\beta)} z^{\beta} J_{0, z}^{\alpha, \beta, \gamma} f(z) ; & 0 \leq \alpha \leq 1 \\ \frac{\Gamma(1+p-\beta) \Gamma(1+p+\gamma-\alpha)}{\Gamma(1+p) \Gamma(1+p+\gamma-\beta)} z^{\beta} \mathrm{I}_{0, z}^{-\alpha, \beta, \gamma} f(z) ; & -\infty \leq \alpha<0\end{cases}
$$

where $J_{0, z}^{\alpha, \beta, \gamma} f(z)$ and $\mathrm{I}_{0, z}^{-\alpha, \beta, \gamma} f(z)$ are, respectively the fractional derivative of $f$ of order $\alpha$ if $0 \leq \alpha<1$ and the fractional integral of $f$ of order $-\alpha$ if $-\infty \leq \alpha<0$.

It is easily verified (see Choi [3]) from (1.9) that

$$
(p-\beta) U_{0, z}^{\alpha+1, \beta+1, \gamma+1} f(z)+\beta U_{0, z}^{\alpha, \beta, \gamma} f(z)=z\left(U_{0, z}^{\alpha, \beta, \gamma} f(z)\right)^{\prime} .
$$

Note that

$$
U_{0, z}^{\alpha, \alpha, \gamma} f(z)=\Omega_{z}^{(\alpha, p)} f(z)(-\infty<\alpha<1)
$$

The fractional differintegral operator $\Omega_{z}^{(\alpha, p)} f(z)$ for $(-\infty<\alpha<p+1)$ is studied by Patel and Mishra [12], and the fractional differential operator $\Omega_{z}^{(\alpha, p)}$ with $0 \leq \alpha<1$ was investigated by Srivastava and Aouf [17]. We, further observe that $\Omega_{z}^{(\alpha, 1)}=\Omega_{z}^{\alpha}$ is the operator introduced and studied by Owa and Srivastava [11].

It is interesting to observe that

$$
U_{0, z}^{0,0, \gamma} f(z)=f(z)
$$

and

$$
U_{0, z}^{1,1, \gamma} f(z)=\frac{z}{p} f^{\prime}(z)
$$

To prove our results, we need the following definitions and lemmas. 
Definition 4 ([7]). Denote by $Q$ the set of all functions $q(z)$ that are analytic and injective on $\bar{U} / E(q)$ where

$$
E(q)=\left\{\zeta \in \partial U: \lim _{z \rightarrow \zeta} q(z)=\infty\right\},
$$

and are such that $q^{\prime}(\zeta) \neq 0$ for $\zeta \in \partial U / E(q)$. Further let the subclass of $Q$ for which $q(0)=a$ be denoted by $Q(a), Q(0) \equiv Q_{0}$ and $Q(1) \equiv Q_{1}$.

Definition 5 ([8]). A function $L(z, t)(z \in U, t \geq 0)$ is said to be a subordination chain if $L(0, t)$ is analytic and univalent in $z \in U$ for all $t \geq 0, L(z, 0)$ is continuously differentiable on [0;1] for all $z \in U$ and $L\left(z, t_{1}\right) \prec L\left(z, t_{2}\right)$ for all $0 \leq t_{1} \leq t_{2}$.

Lemma 1 ([13]). The function $L(z, t): U \times[0 ; 1] \rightarrow \mathbb{C}$ of the form

$$
L(z, t)=a_{1}(t) z+a_{2}(t) z^{2}+\cdots \quad\left(a_{1}(t) \neq 0 ; t \geq 0\right),
$$

and $\lim _{t \rightarrow \infty}\left|a_{1}(t)\right|=\infty$ is a subordination chain if and only if

$$
R e\left\{\frac{z \partial L(z, t) / \partial t}{\partial L(z, t) / \partial t}\right\}>0(z \in U, t \geq 0) .
$$

Lemma 2 ([5]). Suppose that the function $H: \mathbb{C}^{2} \rightarrow \mathbb{C}$ satisfies the condition

$$
\operatorname{Re}\{H(i s ; t)\} \leq 0
$$

for all real s and for all $t \leq-n\left(1+s^{2}\right) / 2, n \in \mathbb{N}$. If the function $p(z)=1+a_{n} z^{n}+a_{n+1} z^{n+1}+\cdots$, is analytic in $U$ and $\operatorname{Re}\left\{H\left(p(z) ; z p^{\prime}(z)\right)\right\}>0(z \in U)$. then $\operatorname{Re}\{p(z)\}>0$ for $z \in U$.

Lemma 3 ([6]). Let $k, \gamma \in \mathbb{C}$ with $k \neq 0$ and let $h \in H(U)$ with $H(0)=c$. If $\operatorname{Re}\{k h(z)+\gamma\}>$ $0(z \in U)$, then the solution of the following differential equation:

$$
q(z)+\frac{z q^{\prime}(z)}{k q(z)+\gamma}=h(z)(z \in U ; q(0)=c),
$$

is analytic in $U$ and satisfies $\operatorname{Re}\{k h(z)+\gamma\}>0$ for $z \in U$.

Lemma 4 ([7]). Let $p \in Q(a)$ and let $q(z)=a+a_{n} z^{n}+a_{n+1} z^{n+1}+\cdots$, be analytic in $U$ with $q(z) \neq 0$ and $n \geq 1$. If $q$ is not subordinate to $p$, the there exists two points $z_{0}=r_{0} e^{i \theta} \in U$ and $\xi_{0} \in \partial U / E(q)$ such that $q\left(U_{r_{0}}\right) \subset p(U) ; q\left(z_{0}\right)=p\left(\xi_{0}\right)$ and $z_{0} p^{\prime}\left(z_{0}\right)=m \xi_{0} p\left(\xi_{0}\right) m \geq n$.

Lemma 5 ([8]). Let $q \in H[a, 1]$ and $\phi: \mathbb{C}^{2} \rightarrow \mathbb{C}$ also $\phi\left(q(z), z q^{\prime}(z)\right)=h(z)$. If $L(z, t)=\phi(q(z)$, $\left.t z q^{\prime}(z)\right)$ is a subordination chain and $q \in H[a, 1] \cap Q(a)$, then

$$
h(z) \prec \phi\left(p(z), z p^{\prime}(z)\right)
$$

implies that $q(z) \prec p(z)$. Further if $\phi\left(q(z), z q^{\prime}(z)\right)=h(z)$ has a univalent solution $q \in Q(a)$, then $q$ is the best subordination. 
In the present paper, we aim to prove some subordination-preserving and superordination -preserving properties associated with the fractional differintegral operator $U_{0, z}^{\alpha, \beta, \gamma}$. Sandwich-type result involving this operator is also derived. A simililar problem for analytic functions was studied by Aouf and Seoudy [1] and [2].

2. Subordination, superordination and sandwich results involving the operator $U_{0, z}^{\alpha, \beta, \gamma}$

Theorem 1. Let $f, g \in A_{p}$ and let

$$
\Re\left\{1+\frac{z \phi^{\prime \prime}(z)}{\phi^{\prime}(z)}\right\}>-\delta
$$

where

$$
\begin{gathered}
\phi(z)=\left(\frac{U_{0, z}^{\alpha+1, \beta+1, \gamma+1} g(z)}{U_{0, z}^{\alpha, \beta, \gamma} g(z)}\right)\left(\frac{U_{0, z}^{\alpha, \beta, \gamma} g(z)}{z^{p}}\right)^{\mu} \\
\left(-\infty<\alpha<1 ;-\infty<\beta<1 ; \gamma \in \mathbb{R}^{+} ; \mu>0 ; z \in U\right),
\end{gathered}
$$

and $\delta$ is given by

$$
\delta=\frac{1+\mu^{2}(p-\beta)^{2}-\left|1-\mu^{2}(p-\beta)^{2}\right|}{4 \mu(p-\beta)} .
$$

Then the subordination condition

$$
\left(\frac{U_{0, z}^{\alpha+1, \beta+1, \gamma+1} f(z)}{U_{0, z}^{\alpha, \beta, \gamma} f(z)}\right)\left(\frac{U_{0, z}^{\alpha, \beta, \gamma} f(z)}{z^{p}}\right)^{\mu}<\left(\frac{U_{0, z}^{\alpha+1, \beta+1, \gamma+1} g(z)}{U_{0, z}^{\alpha, \beta, \gamma} g(z)}\right)\left(\frac{U_{0, z}^{\alpha, \beta, \gamma} g(z)}{z^{p}}\right)^{\mu},
$$

implies that

$$
\left(\frac{U_{0, z}^{\alpha, \beta, \gamma} f(z)}{z^{p}}\right)^{\mu}<\left(\frac{U_{0, z}^{\alpha, \beta, \gamma} g(z)}{z^{p}}\right)^{\mu}
$$

and the function $\left(\frac{U_{0, z}^{\alpha, \beta, \gamma} g(z)}{z^{p}}\right)^{\mu}$ is the best dominant.

Proof. Let us define the functions $F(z)$ and $G(z)$ in $U$ by

$$
F(z)=\left(\frac{U_{0, z}^{\alpha, \beta, \gamma} f(z)}{z^{p}}\right)^{\mu} \quad \text { and } \quad G(z)=\left(\frac{U_{0, z}^{\alpha, \beta, \gamma} g(z)}{z^{p}}\right)^{\mu}(z \in U),
$$

we assume here, without loss of generality, that $G(z)$ is analytic and univalent on $\bar{U}$ and

$$
G^{\prime}(\zeta) \neq 0 \quad(|\zeta|=1)
$$


If not, then we replace $F(z)$ and $G(z)$ by $F(\rho z)$ and $G(\rho z)$, respectively, with $0<\rho<1$. These new functions have the desired properties on $\bar{U}$, and we can use them in the proof of our result. Therefore, the results would follow by letting $\rho \rightarrow 1$. We first show that, if

$$
q(z)=1+\frac{z G^{\prime \prime}(z)}{G^{\prime}(z)} \quad(z \in U)
$$

then

$$
\Re\{q(z)\}>0(z \in U) .
$$

From (1.11) and the definition of the functions $G, \phi$, we obtain that

$$
\phi(z)=G(z)+\frac{z G^{\prime}(z)}{\mu(p-\beta)} .
$$

Differentiating both side of (2.6) with respect to $z$ yields

$$
\phi^{\prime}(z)=\left(1+\frac{1}{\mu(p-\beta)}\right) G^{\prime}(z)+\frac{z G^{\prime}(z)}{\mu(p-\beta)} .
$$

Combining (2.5) and (2.7), we easily get

$$
1+\frac{z \phi^{\prime \prime}(z)}{\phi^{\prime}(z)}=q(z)+\frac{z q^{\prime}(z)}{q(z)+\mu(p-\beta)}=h(z) \quad(z \in U) .
$$

It follows from (2.1) and (2.8) that

$$
\operatorname{Re}\{h(z)+\mu(p-\beta)\}>0(z \in U) .
$$

Moreover, by using Lemma 3, we conclude that the differential equation (2.8) has a solution $q(z) \in H(U)$ with $h(0)=q(0)=1$. Let

$$
H(u, v)=u+\frac{v}{u+\mu(p-\beta)}+\delta,
$$

where $\delta$ is given by (2.3). From (2.8) and (2.9), we obtain

$$
R e\left\{H\left(q(z) ; z q^{\prime}(z)\right)\right\}>0(z \in U) .
$$

To verify the condition that

$$
R e\{H(i s ; t)\} \leq 0 \quad\left(t \leq-\left(1+s^{2}\right) / 2 ; s \in \mathbb{R}\right) .
$$

we proceed it as follows:

$$
\operatorname{Re}\{H(i s ; t)\}=\operatorname{Re}\left\{i s+\frac{t}{i s+\mu(p-\beta)}+\delta\right\}=\frac{t \mu(p-\beta)}{s^{2}+\mu^{2}(p-\beta)^{2}}+\delta
$$




$$
\leq-\frac{\psi_{p}(\beta, \mu, \delta, s)}{2\left[s^{2}+\mu^{2}(p-\beta)^{2}\right]}
$$

where

$$
\psi_{p}(\beta, \mu, \delta, s)=[\mu(p-\beta)-2 \delta] s^{2}-2 \delta \mu^{2}(p-\beta)^{2}+\mu(p-\beta) .
$$

For $\delta$ given by (2.3), we note that the expression $\psi_{p}(\beta, \mu, \delta, s)$ in (2.11) is a positive, which implies that (2.10) holds. Thus, by using Lemma 2, we conclude that

$$
\operatorname{Re}\{q(z)\}>0(z \in U) .
$$

By the definition of $q(z)$, we know that $G$ is convex. To prove $F \prec G$, let the function $L(z, t)$ be defined by

$$
L(z, t)=G(z)+\frac{(1+t) z G^{\prime}(z)}{\mu(p-\beta)} \quad(0 \leq t<\infty ; z \in U) .
$$

Since $G$ is convex, then

$$
\left.\frac{\partial L(z, t)}{\partial z}\right|_{z=0}=G^{\prime}(0)\left(1+\frac{(1+t)}{\mu(p-\beta)}\right) \neq 0 \quad(0 \leq t<\infty ; z \in U)
$$

and

$$
\operatorname{Re}\left\{\frac{z \partial L(z, t) / \partial t}{\partial L(z, t) / \partial t}\right\}=\operatorname{Re}\{\mu(p-\beta)+(1+t) q(z)\}>0(0 \leq t<\infty ; z \in U) .
$$

Therefore, by using Lemma 1, we deduce that $L(z, t)$ is a subordination chain. It follows from the definition of subordination chain that

$$
\phi(z)=G(z)+\frac{z G^{\prime}(z)}{\mu(p-\beta)}=L(z, 0),
$$

and

$$
L(z, 0) \prec L(z, t) \quad(0 \leq t<\infty)
$$

which implies

$$
L(\zeta, t) \notin L(U, 0) \quad(0 \leq t<\infty ; \zeta \in \partial U),
$$

If $F$ is not subordinate to $G$, by using Lemma 4, we know that there exist two points $z_{0} \in U$ and $\zeta_{0} \in \partial U$ such that

$$
F\left(z_{0}\right)=G\left(\zeta_{0}\right) \operatorname{and} z_{0} F^{\prime}\left(z_{0}\right)=(1+t) \zeta_{0} p\left(\zeta_{0}\right)(0 \leq t<\infty)
$$

Hence, by virtue of (1.11) and (2.14), we have

$$
\begin{aligned}
L\left(\zeta_{0}, t\right) & =G\left(\zeta_{0}\right)+\frac{(1+t) z G^{\prime}\left(\zeta_{0}\right)}{\mu(p-\beta)}=F\left(z_{0}\right)+\frac{z_{0} F^{\prime}\left(z_{0}\right)}{\mu(p-\beta)} \\
& =\left(\frac{U_{0, z}^{\alpha+1, \beta+1, \gamma+1} f\left(z_{0}\right)}{U_{0, z}^{\alpha, \beta, \gamma} f\left(z_{0}\right)}\right)\left(\frac{U_{0, z}^{\alpha, \beta, \gamma} f\left(z_{0}\right)}{z_{0}^{p}}\right)^{\mu} \in \phi(U) .
\end{aligned}
$$


This contradicts to (2.13). Thus, we deduce that $F \prec G$. Considering $F=G$, we see that the function $G$ is the best dominant. This completes the proof of Theorem 1.

By taking $\alpha=\beta$ in Theorem 1 and using the relation (1.12) we get the following Corollary

Corollary 1. Let $f, g \in A_{p}$ and let

$$
\Re\left\{1+\frac{z \phi^{\prime \prime}(z)}{\phi^{\prime}(z)}\right\}>-\delta,
$$

where

$$
\phi(z)=\left(\frac{\Omega_{z}^{(\alpha+1, p)} g(z)}{\Omega_{z}^{(\alpha, p)} g(z)}\right)\left(\frac{\Omega_{z}^{(\alpha, p)} g(z)}{z^{p}}\right)^{\mu}(-\infty<\alpha<1 ; \mu>0 ; z \in U),
$$

and $\delta$ is given by

$$
\delta=\frac{1+\mu^{2}(p-\alpha)^{2}-\left|1-\mu^{2}(p-\alpha)^{2}\right|}{4 \mu(p-\alpha)} .
$$

Then the subordination condition

$$
\left(\frac{\Omega_{z}^{(\alpha+1, p)} f(z)}{\Omega_{z}^{(\alpha, p)} f(z)}\right)\left(\frac{\Omega_{z}^{(\alpha, p)} f(z)}{z^{p}}\right)^{\mu}<\left(\frac{\Omega_{z}^{(\alpha+1, p)} g(z)}{\Omega_{z}^{(\alpha, p)} g(z)}\right)\left(\frac{\Omega_{z}^{(\alpha, p)} g(z)}{z^{p}}\right)^{\mu},
$$

implies that $\left(\frac{\Omega_{z}^{(\alpha, p)} f(z)}{z^{p}}\right)^{\mu}<\left(\frac{\Omega_{z}^{(\alpha, p)} g(z)}{z^{p}}\right)^{\mu}$, and the function $\left(\frac{\Omega_{z}^{(\alpha, p)} g(z)}{z^{p}}\right)^{\mu}$ is the best dominant.

By taking $\alpha=0$ in Corollary 1 and using the relation (1.13) and (1.14) we get the following Corollary

Corollary 2. Let $f, g \in A_{p}$ and let

$$
\Re\left\{1+\frac{z \phi^{\prime \prime}(z)}{\phi^{\prime}(z)}\right\}>-\delta,
$$

where

$$
\phi(z)=\left(\frac{z g^{\prime}(z)}{p g(z)}\right)\left(\frac{g(z)}{z^{p}}\right)^{\mu}(\mu>0 ; z \in U)
$$

and $\delta$ is given by

$$
\delta=\frac{1+\mu^{2} p^{2}-\left|1-\mu^{2} p^{2}\right|}{4 \mu p} .
$$

Then the subordination condition

$$
\left(\frac{z f^{\prime}(z)}{p f(z)}\right)\left(\frac{f(z)}{z^{p}}\right)^{\mu} \prec\left(\frac{z g^{\prime}(z)}{p g(z)}\right)\left(\frac{g(z)}{z^{p}}\right)^{\mu},
$$

implies that

$$
\left(\frac{f(z)}{z^{p}}\right)^{\mu} \prec\left(\frac{g(z)}{z^{p}}\right)^{\mu},
$$

and the function $\left(\frac{g(z)}{z^{p}}\right)^{\mu}$ is the best dominant. 
We now derive the following superordination result.

Theorem 2. Let $f, g \in A_{p}$ and let

$$
\Re\left\{1+\frac{z \phi^{\prime \prime}(z)}{\phi^{\prime}(z)}\right\}>-\delta
$$

where

$$
\begin{gathered}
\phi(z)=\left(\frac{U_{0, z}^{\alpha+1, \beta+1, \gamma+1} g(z)}{U_{0, z}^{\alpha, \beta, \gamma} g(z)}\right)\left(\frac{U_{0, z}^{\alpha, \beta, \gamma} g(z)}{z^{p}}\right)^{\mu} \\
\left(-\infty<\alpha<1 ;-\infty<\beta<1 ; \gamma \in \mathbb{R}^{+} ; \mu>0 ; z \in U\right),
\end{gathered}
$$

and $\delta$ is given by (2.3). If the function $\left(\frac{U_{0, z}^{\alpha+1, \beta+1, \gamma+1} f(z)}{U_{0, z}^{\alpha, \beta, \gamma} f(z)}\right)\left(\frac{U_{0, z}^{\alpha, \beta, \gamma} f(z)}{z^{p}}\right)^{\mu}$ is univalent in $U$ and $\left(\frac{U_{0, z}^{\alpha, \beta, \gamma} f(z)}{z^{p}}\right)^{\mu} \in Q$, then the superordination condition

$$
\left(\frac{U_{0, z}^{\alpha+1, \beta+1, \gamma+1} g(z)}{U_{0, z}^{\alpha, \beta, \gamma} g(z)}\right)\left(\frac{U_{0, z}^{\alpha, \beta, \gamma} g(z)}{z^{p}}\right)^{\mu} \prec\left(\frac{U_{0, z}^{\alpha+1, \beta+1, \gamma+1} f(z)}{U_{0, z}^{\alpha, \beta, \gamma} f(z)}\right)\left(\frac{U_{0, z}^{\alpha, \beta, \gamma} f(z)}{z^{p}}\right)^{\mu},
$$

implies that

$$
\left(\frac{U_{0, z}^{\alpha, \beta, \gamma} g(z)}{z^{p}}\right)^{\mu} \prec\left(\frac{U_{0, z}^{\alpha, \beta, \gamma} f(z)}{z^{p}}\right)^{\mu}
$$

and the function $\left(\frac{U_{0, z}^{\alpha, \beta, \gamma} g(z)}{z^{p}}\right)^{\mu}$ is the best subordinan.

Proof. Suppose that the functions $F, G$ and $q$ are defined by (2.4) and (2.5), respectively. By applying the similar method as in the proof of Theorem 1, we get

$$
\operatorname{Re}\{q(z)\}>0(z \in U) .
$$

Next, to arrive at our desired result, we show that $G \prec F$. For this, we suppose that the function $L(z, t)$ be defined by (2.12).

Since $G$ is convex, by applying a similar method as in Theorem 1 , we deduce that $L(z, t)$ is subordination chain. Therefore, by using Lemma 5, we conclude that $G \prec F$. Moreover, since the differential equation

$$
\phi(z)=G(z)+\frac{z G^{\prime}(z)}{\mu(p-\beta)}=\varphi\left(G(z), z G^{\prime}(z)\right)
$$

has a univalent solution $G$, it is the best subordinant. This completes the proof.

By taking $\alpha=\beta$ in Theorem 2 and using the relation (1.12) we get the following Corollary 
Corollary 3. Let $f, g \in A_{p}$ and let

$$
\Re\left\{1+\frac{z \phi^{\prime \prime}(z)}{\phi^{\prime}(z)}\right\}>-\delta
$$

where

$$
\phi(z)=\left(\frac{\Omega_{z}^{(\alpha+1, p)} g(z)}{\Omega_{z}^{(\alpha, p)} g(z)}\right)\left(\frac{\Omega_{z}^{(\alpha, p)} g(z)}{z^{p}}\right)^{\mu}(-\infty<\alpha<1 ; \mu>0 ; z \in U),
$$

and $\delta$ is given by (2.3). If the function $\left(\frac{\Omega_{z}^{(\alpha+1, p)} g(z)}{\Omega_{z}^{(\alpha, p)} g(z)}\right)\left(\frac{\Omega_{z}^{(\alpha, p)} g(z)}{z^{p}}\right)^{\mu}$ is univalent in $U$ and $\left(\frac{\Omega_{z}^{(\alpha, p)} g(z)}{z^{p}}\right)^{\mu}$ $\in Q$, then the superordination condition

$$
\left(\frac{\Omega_{z}^{(\alpha+1, p)} g(z)}{\Omega_{z}^{(\alpha, p)} g(z)}\right)\left(\frac{\Omega_{z}^{(\alpha, p)} g(z)}{z^{p}}\right)^{\mu}<\left(\frac{\Omega_{z}^{(\alpha+1, p)} f(z)}{\Omega_{z}^{(\alpha, p)} f(z)}\right)\left(\frac{\Omega_{z}^{(\alpha, p)} f(z)}{z^{p}}\right)^{\mu},
$$

implies that

$$
\left(\frac{\Omega_{z}^{(\alpha, p)} g(z)}{z^{p}}\right)^{\mu}<\left(\frac{\Omega_{z}^{(\alpha, p)} f(z)}{z^{p}}\right)^{\mu},
$$

and the function $\left(\frac{\Omega_{z}^{(\alpha, p)} g(z)}{z^{p}}\right)^{\mu}$ is the best subordinant.

By taking $\alpha=0$ in Corollary 3 and using the relation (1.13) and (1.14) we get the following corollary

Corollary 4. Let $f, g \in A_{p}$ and let

$$
\Re\left\{1+\frac{z \phi^{\prime \prime}(z)}{\phi^{\prime}(z)}\right\}>-\delta
$$

where

$$
\phi(z)=\left(\frac{z g^{\prime}(z)}{p g(z)}\right)\left(\frac{g(z)}{z^{p}}\right)^{\mu}(\mu>0 ; z \in U),
$$

and $\delta$ is given by (2.3). If the function $\left(\frac{z g^{\prime}(z)}{p g(z)}\right)\left(\frac{g(z)}{z^{p}}\right)^{\mu}$ is univalent in $U$ and $\left(\frac{g(z)}{z^{p}}\right)^{\mu} \in Q$, then the superordination condition

$$
\left(\frac{z g^{\prime}(z)}{p g(z)}\right)\left(\frac{g(z)}{z^{p}}\right)^{\mu}<\left(\frac{z f^{\prime \prime}(z)}{p f(z)}\right)\left(\frac{f(z)}{z^{p}}\right)^{\mu},
$$

implies that

$$
\left(\frac{g(z)}{z^{p}}\right)^{\mu} \prec\left(\frac{f(z)}{z^{p}}\right)^{\mu},
$$

and the function $\left(\frac{g(z)}{z^{p}}\right)^{\mu}$ is the best dominant.

Combining Theorems 1 and 2, we obtain the following "sandwich-type result". 
Theorem 3. Let $f, g_{i} \in A_{p}(j=1,2)$ and let

$$
\Re\left\{1+\frac{z \phi_{j}^{\prime \prime}(z)}{\phi_{j}^{\prime}(z)}\right\}>-\delta
$$

where

$$
\begin{aligned}
& \phi_{j}(z)=\left(\frac{U_{0, z}^{\alpha+1, \beta+1, \gamma+1} g_{j}(z)}{U_{0, z}^{\alpha, \beta, \gamma} g_{j}(z)}\right)\left(\frac{U_{0, z}^{\alpha, \beta, \gamma} g_{j}(z)}{z^{p}}\right)^{\mu} \\
& \left(-\infty<\alpha<1 ;-\infty<\beta<1 ; \gamma \in \mathbb{R}^{+} ; \mu>0 ; z \in U\right),
\end{aligned}
$$

and $\delta$ is given by (2.3). If the function $\left(\frac{U_{0, z}^{\alpha+1, \beta+1, \gamma+1} f(z)}{U_{0, z}^{\alpha, \beta, \gamma} f(z)}\right)\left(\frac{U_{0, z}^{\alpha, \beta, \gamma} f(z)}{z^{p}}\right)^{\mu}$ is univalent in $U$ and $\left(\frac{U_{0, z}^{\alpha, \beta, \gamma} f(z)}{z^{p}}\right)^{\mu} \in Q$, then the condition

$$
\begin{aligned}
\left(\frac{U_{0, z}^{\alpha+1, \beta+1, \gamma+1} g_{1}(z)}{U_{0, z}^{\alpha, \beta, \gamma} g_{1}(z)}\right)\left(\frac{U_{0, z}^{\alpha, \beta, \gamma} g_{1}(z)}{z^{p}}\right)^{\mu} & <\left(\frac{U_{0, z}^{\alpha+1, \beta+1, \gamma+1} f(z)}{U_{0, z}^{\alpha, \beta, \gamma} f(z)}\right)\left(\frac{U_{0, z}^{\alpha, \beta, \gamma} f(z)}{z^{p}}\right)^{\mu} \\
& \prec\left(\frac{U_{0, z}^{\alpha+1, \beta+1, \gamma+1} g_{2}(z)}{U_{0, z}^{\alpha, \beta, \gamma} g_{2}(z)}\right)\left(\frac{U_{0, z}^{\alpha, \beta, \gamma} g_{2}(z)}{z^{p}}\right)^{\mu},
\end{aligned}
$$

implies that

$$
\left(\frac{U_{0, z}^{\alpha, \beta, \gamma} g_{1}(z)}{z^{p}}\right)^{\mu} \prec\left(\frac{U_{0, z}^{\alpha, \beta, \gamma} f(z)}{z^{p}}\right)^{\mu} \prec\left(\frac{U_{0, z}^{\alpha, \beta, \gamma} g_{1}(z)}{z^{p}}\right)^{\mu},
$$

and the function $\left(\frac{U_{0, z}^{\alpha, \beta, \gamma} g_{1}(z)}{z^{p}}\right)^{\mu}$ and $\left(\frac{U_{0, z}^{\alpha, \beta, \gamma} g_{2}(z)}{z^{p}}\right)^{\mu}$ are, respectively, the best subordinant and the best dominant.

By taking $\alpha=\beta$ in Theorem 3 and using the relation (1.12) we get the following Corollary Corollary 5. Let $f, g_{i} \in A_{p}(j=1,2)$ and let

$$
\Re\left\{1+\frac{z \phi_{j}^{\prime \prime}(z)}{\phi_{j}^{\prime}(z)}\right\}>-\delta,
$$

where

$$
\phi_{j}(z)=\left(\frac{\Omega_{z}^{(\alpha+1, p)} g_{j}(z)}{\Omega_{z}^{(\alpha, p)} g_{j}(z)}\right)\left(\frac{\Omega_{z}^{(\alpha, p)} g_{j}(z)}{z^{p}}\right)^{\mu}(-\infty<\alpha<1 ; \mu>0 ; z \in U),
$$

and $\delta$ is given by (2.3). If the function $\left(\frac{\Omega_{z}^{(\alpha+1, p)} f(z)}{\Omega_{z}^{(\alpha, p)} f(z)}\right)\left(\frac{\Omega_{z}^{(\alpha, p)} f(z)}{z^{p}}\right)^{\mu}$ is univalent in $U$ and $\left(\frac{\Omega_{z}^{(\alpha, p)} f(z)}{z^{p}}\right)^{\mu}$ $\in Q$, then the condition

$$
\left(\frac{\Omega_{z}^{(\alpha+1, p)} g_{1}(z)}{\Omega_{z}^{(\alpha, p)} g_{1}(z)}\right)\left(\frac{\Omega_{z}^{(\alpha, p)} g_{1}(z)}{z^{p}}\right)^{\mu}<\left(\frac{\Omega_{z}^{(\alpha+1, p)} f(z)}{\Omega_{z}^{(\alpha, p)} f(z)}\right)\left(\frac{\Omega_{z}^{(\alpha, p)} f(z)}{z^{p}}\right)^{\mu}
$$




$$
\prec\left(\frac{\Omega_{z}^{(\alpha+1, p)} g_{2}(z)}{\Omega_{z}^{(\alpha, p)} g_{2}(z)}\right)\left(\frac{\Omega_{z}^{(\alpha, p)} g_{2}(z)}{z^{p}}\right)^{\mu},
$$

implies that

$$
\left(\frac{\Omega_{z}^{(\alpha, p)} g_{1}(z)}{z^{p}}\right)^{\mu} \prec\left(\frac{\Omega_{z}^{(\alpha, p)} f(z)}{z^{p}}\right)^{\mu} \prec\left(\frac{\Omega_{z}^{(\alpha, p)} g_{1}(z)}{z^{p}}\right)^{\mu},
$$

and the function $\left(\frac{\Omega_{z}^{(\alpha, p)} g_{1}(z)}{z^{p}}\right)^{\mu}$ and $\left(\frac{\Omega_{z}^{(\alpha, p)} g_{2}(z)}{z^{p}}\right)^{\mu}$ are, respectively, the best subordinant and the best dominant.

By taking $\alpha=0$ in Corollary 5 and using the relation (1.13) and (1.14) we get the following Corollary.

Corollary 6. Let $f, g_{i} \in A_{p}(j=1,2)$ and let

$$
\Re\left\{1+\frac{z \phi_{j}^{\prime \prime}(z)}{\phi_{j}^{\prime}(z)}\right\}>-\delta,
$$

where

$$
\phi_{j}(z)=\left(\frac{z g_{j}^{\prime}(z)}{p g_{j}(z)}\right)\left(\frac{g_{j}(z)}{z^{p}}\right)^{\mu}(\mu>0 ; z \in U),
$$

and $\delta$ is given by (2.3). If the function $\left(\frac{z f^{\prime}(z)}{p f(z)}\right)\left(\frac{f(z)}{z^{p}}\right)^{\mu}$ is univalent in $U$ and $\left(\frac{f(z)}{z^{p}}\right)^{\mu} \in Q$, then the condition

$$
\left(\frac{z g_{1}^{\prime}(z)}{p g_{1}(z)}\right)\left(\frac{g_{1}(z)}{z^{p}}\right)^{\mu} \prec\left(\frac{z f^{\prime}(z)}{p f(z)}\right)\left(\frac{f(z)}{z^{p}}\right)^{\mu} \prec\left(\frac{z g_{2}^{\prime}(z)}{p g_{2}(z)}\right)\left(\frac{g_{2}(z)}{z^{p}}\right)^{\mu},
$$

implies that

$$
\left(\frac{g_{1}(z)}{z^{p}}\right)^{\mu}<\left(\frac{f(z)}{z^{p}}\right)^{\mu} \prec\left(\frac{g_{1}(z)}{z^{p}}\right)^{\mu},
$$

and the function $\left(\frac{g_{1}(z)}{z^{p}}\right)^{\mu}$ and $\left(\frac{g_{2}(z)}{z^{p}}\right)^{\mu}$ are, respectively, the best subordinant and the best dominant.

\section{Acknowledgement}

The author would like to express many thanks to the referee for his valuable and constructive suggestions. 


\section{References}

[1] M. K. Aouf and T. M. Seoudy, Some preserving subordination and superordination of the Liu-Owa integral operator, Complex Anal. Oper. Theory, in press (doi:10.1007/s11785-011-0141-6).

[2] M. K. Aouf and T. M. Seoudy, Some preserving subordination and superordination of analytic functions involving the Liu-Owa integral operator, Comput. Math. Appl. 62(2011), 3575-3580.

[3] J. H. Choi, On differential subordinations of multivalent functions involving a certain fractional derivative operator, Int. J. Math. Math. Sci., doi: 10.1155/2010/952036.

[4] S. M. Khainar and M. More, A subclass of uniformly convex functions associated with certain fractional calculus operator, IAENG International Journal of Applied Mathematics, 39(3) (2009), IJAM-39-07.

[5] S. S. Miller and P. T. Mocanu, Differential subordinations and univalent functions, Michigan Math. J., 28(2)(1981), 157-172.

[6] S. S. Miller and P.T. Mocanu, Univalent solutions of Briot-Bouquet differential equations, J. Differential Equations, 56(3) (1985), 297-309.

[7] S. S. Miller and P. T. Mocanu, Differential Subordinations: Theory and Applications, in: Monographs and Textbooks in Pure and Applied Mathematics, vol. 225, Marcel Dekker, StateNew York, CityplaceBasel, 2000

[8] S. S. Miller and P. T. Mocanu, Subordinants of differential superordinations, Complex Var. Theory Appl., 48(10) (2003), 815-826.

[9] G. Murugusundaramoorthy, T. Rosy and M. Darus, A subclass of uniformly convex functions associated with certain fractional calculus operators, J. Inequal. Pure and Appl. Math., 6(3)(2005), Article 86, [online:http: \jipam.vu.edu.au].

[10] S. Owa, On the distortion theorems I, Kyungpook Math. J., 18(1978), 53-59.

[11] S. Owa and H. M. Srivastava, Univalent and starlike generalized hypergeometric function, Canad. J. Math., 39(1987), 1057-1077.

[12] J. Patel and A. K. Mishra, On certain subclasses of multivalent functions associated with an extended fractional differintegral operator, J. Math. Anal. Appl., 332(2007), 109-122.

[13] C. Pommerenke, Univalent Functions, Vandenhoeck and Ruprecht, Götingen, 1975.

[14] M. Saigo, A certain boundary value problem for the Euler-Darboux equation, Math. Apon., 25(1979), 377-385.

[15] M. Saigo, A remark on integral operators involving the Gauss hypergeometric functions, Math. Rep. Kyushu Univ. 11(1978), 135-143.

[16] H. M. Srivastava and S. Owa (Eds.), Univalent Functions, Fractional Calculus, and Their Applications, Halsted Press(Ellis Horwood Limited, Chichester), John Wiley and Sons, placeStateNew York, 1989.

[17] H. M. Srivastava and M. K. Aouf, A certain fractional derivative operator and its applications to a new class of analytic and multivalent functions with negative coefficients I, J. Math. Anal. Appl., 171(1992) 1-13.

Department of Mathematics, Alazhar University-Gaza, P. O. Box 1277, Gaza, Palestine.

E-mail: shenanjm @yahoo.com 